\title{
Online Identification of Time-Variant Structural Parameters Under Unknown Inputs Basing On Extended Kalman Filter
}

\section{Xiaoxiong Zhang}

Hunan University

Jia He ( $\nabla$ jiahe@hnu.edu.cn )

Hunan University

\section{Xugang Hua}

Hunan University

\section{Zhengqing Chen}

Hunan University

\section{Ou Yang}

Hunan University

\section{Research Article}

Keywords: extended Kalman filter, time-varying parameters identification, unknown loads, revised observation equation, online tracking matrix

Posted Date: December 8th, 2021

DOI: https://doi.org/10.21203/rs.3.rs-1106079/v1

License: (c) (i) This work is licensed under a Creative Commons Attribution 4.0 International License. Read Full License 


\title{
Online identification of time-variant structural parameters under unknown inputs basing on extended Kalman filter
}

\author{
Xiaoxiong Zhang, Jia He*, Xugang Hua, Zhengqing Chen, Ou Yang \\ Key laboratory of wind and bridge engineering of Hunan Province, Key laboratory of \\ Building Safety and Energy Efficiency of the Ministry of Education, Hunan University, \\ Changsha, China
}

* Corresponding author: jiahe@ hnu.edu.cn

\begin{abstract}
To date, a number of parameter identification methods have been developed for the purpose of structural health monitoring and vibration control. Among them, the extended Kalman filter (EKF) series methods are attractive in view of the efficient unbiased estimation in recursive manner. However, most of these methods are performed on the premise that the parameters are time-invariant and/or the loadings are known. To circumvent the aforementioned limitations, an online EKF with unknown input (OEKF-UI) approach is proposed in this paper for the identification of time-varying parameters and the unknown excitation. A revised observation equation is obtained with the aid of projection matrix. To capture the changes of structural parameters in realtime, an online tracking matrix (OTM) associated with the time-varying parameters is introduced and determined via an optimization procedure. Then, based on the principle of EKF, the recursive solution of structural states including the time-variant parameters can be analytically derived. Finally, using the estimated structural states, the unknown inputs are identified by means of least-squares estimation (LSE) at the same time-step. The effectiveness of the proposed approach is validated via linear and nonlinear numerical examples with the consideration of parameters being varied abruptly.
\end{abstract}

Keywords: extended Kalman filter, time-varying parameters identification, unknown loads, revised observation equation, online tracking matrix

\section{Introduction}

Owning to severe loadings or other environmental effects, structural damage may occur and accumulate during the service life of structures. In general, the damage can be reflected by the changes of structural parameters, such as stiffness degradation. In this regards, identification of these time-variant parameters plays an important role for structural health assessment and vibration control. To date, a number of parameter identification and damage detection methods have been developed in time domain, frequency domain, or time-frequency domain [1-3]. Among them, the extended Kalman filter (EKF) technique has received considerable attention in view of its computational efficiency, unbiased estimation and recursive manner. 
The effort on the development of EKF-based methods for parameter identification has been made for many years. The earlier contribution can be traced back to the work by Yun and Shinozuka [4]. Since then, increasing attention has been paid for the purpose of health monitoring of civil infrastructures using EKF [5-8]. In the framework of classic EKF, the external excitation should be known for the identification. However, in many situations, the external forces applied to the structures are unmeasurable or difficult to be measured. Therefore, many endeavors have been made to deal with the case of unknown external forces, saying EKF under unknown inputs (EKF-UI). For example, with the sequent usage of LSE and EKF, Lei et al. [9] proposed an EKF-UI approach for damage detection of linear and nonlinear structures. Based on weighted global iteration and a force updated procedure, $\mathrm{Xu}$ and He [10] proposed an EKF-UI approach for substructural identification. Pan et al. [11] proposed a general EKF-UI approach to avoid the restrictions on sensor deployment. By using modal transformation, Liu et al. [12] proposed a modal EKF-UI approach for the identification of large-scale structures. By treating the nonlinear restoring force as unknown fictitious inputs, Lei et al. [13] proposed an approach for nonlinear identification without the prior knowledge of the nonlinear model. Using perturbation analysis, Impraimakis and Smyth [14] proposed an unscented Kalman filter method for joint estimation of structural parameters and external excitation.

The parameters to be identified in EKF algorithm are all considered as a part of the extended state vector. In many existing EKF-based methods, these parameters are assumed to be time-independent such that their time derivative would be zero. However, due to severe loadings or other environmental effects, physical parameters usually vary during the service life of structures. Thus, it is highly desirable to develop effective methods to accurately identify the time-varying parameters and adaptively evaluate the structural performance. Much effort has been made in this field basing on various optimization algorithms, such as least squares estimation [15-17], wavelet transformation [18, 19], Hilbert transformation [20], neural networks [21]. It is not intended to give a comprehensive review on the time-varying parameters identification methods in this paper. Instead, some investigation based on Kalman filter $(\mathrm{KF})$ principle are briefly introduced herein. For example, the performance of adaptive fading EKF for the identification of time-variant frame structures were discussed by Loh et al. [22]. An EKF-based approach was proposed by Yang et al. [23, 24] to adaptively capture the time-varying parameters with the consideration of known inputs. The effectiveness of this approach was further experimentally validated via a linear and nonlinear building structure $[25,26]$. Li et al. [27] proposed an approach to identify the parameters by combining a time- 
varying forgetting factor stochastic gradient and KF algorithm. Based on the synthesis of wavelet multiresolution decomposition and KF, Chen et al. [28] proposed an approach for the identification of time-varying structural stiffness and damping parameters. This approach was then extended for dealing with the case of unknown inputs [29, 30]. An adaptive EKF with two computational modes was proposed by Yang et al. [31] for time-varying parameter identification under seismic excitations. Huang et al. [32] proposed a dual KF-based approach for real-time Bayesian sequential state and parameter identification. A generalized EKF-based approach was proposed by Lei et al. [33] for the integration of identification and vibration control of time-varying structures under seismic inputs.

Recently, by using a revised observation equation, the authors proposed an EKF-UI approach for the simultaneous identification of structural parameters and unknown loadings [34]. This approach was then used for substructural identification [35]. However, this approach is performed under the premise that the parameters are constant. In this paper, to circumvent this limitation, an online EKF-UI (OEKF-UI) approach is proposed for the timevarying parametric identification with unknown input information. With the aid of projection matrix, a revised observation equation is derived. An online tracking matrix (OTM), which is used for capturing the time-varying properties of parameters, is defined and determined by an optimization procedure. Then, based on the principle of EKF, the recursive solution of structural states including the time-variant parameters can be analytically derived. Finally, using the estimated structural states, the unknown inputs are identified by means of leastsquares estimation (LSE) at the same time-step. The effectiveness of the proposed approach is numerically validated via several linear and nonlinear examples.

\section{The proposed OEKF-UI approach}

\subsection{Brief introduction of the previously proposed EKF-UI approach}

The authors recently proposed an EKF-UI approach for the parameters and loads identification [34]. Here, a brief introduction of this approach is given.

The second-order differential equation of motion of an $n$ degrees-of-freedom (DOFs) structure can be written as

$$
\mathbf{M} \ddot{\mathbf{x}}(t)+\mathbf{F}[\dot{\mathbf{x}}(t), \mathbf{x}(t), \boldsymbol{\theta}(t)]=\boldsymbol{\varphi}^{u} \mathbf{f}^{u}(t)
$$

where $\ddot{\mathbf{x}}(t), \dot{\mathbf{x}}(t)$ and $\mathbf{x}(\mathrm{t})$ are the vectors of structural acceleration, velocity and displacement, respectively; $\mathbf{M}$ is the mass matrix; $\mathbf{F}[\dot{\mathbf{x}}(t), \mathbf{x}(t), \boldsymbol{\theta}(t)]$ represents the linear or nonlinear 
restoring force vector; $\boldsymbol{\theta}(t)$ is the $m$-dimensional vector of parameters to be identified; $\mathbf{f}^{u}(t)$ is the unknown input and $\varphi^{u}$ is its influence matrix.

Consider an extended state vector,

$$
\mathbf{Z}(t)=\left[\mathbf{x}(t)^{\mathrm{T}}, \dot{\mathbf{x}}(t)^{\mathrm{T}}, \boldsymbol{\theta}(t)^{\mathrm{T}}\right]^{\mathrm{T}}
$$

If the structural parameters are time-invariant, the following equation can be obtained,

$$
\frac{d \mathbf{Z}(t)}{d t}=\left[\begin{array}{c}
\dot{\mathbf{x}}(t) \\
\mathbf{M}^{-1}\left(\mathbf{F}[\dot{\mathbf{x}}(t), \mathbf{x}(t), \boldsymbol{\theta}]+\boldsymbol{\varphi}^{u} \mathbf{f}^{u}(t)\right) \\
\mathbf{0}
\end{array}\right]=\mathbf{u}\left(\mathbf{Z}(t), \mathbf{f}^{u}(t), t\right)+\mathbf{w}(t)
$$

where $\mathbf{w}(t)$ is process noise vector with zero mean and a covariance matrix $\mathbf{Q}(t)$.

Based on the measured acceleration, the discretized observation equation at time instant $t_{k}$ can be described as,

$$
\mathbf{y}_{k}=-\mathbf{M}^{-1} \mathbf{F}\left[\dot{\mathbf{x}}_{k}, \mathbf{x}_{k}, \boldsymbol{\theta}\right]+\mathbf{M}^{-1} \boldsymbol{\varphi} \mathbf{f}_{k}^{u}+\mathbf{v}_{k}=\mathbf{h}\left(\mathbf{Z}_{k}\right)-\mathbf{D} \mathbf{f}_{k}^{u}+\mathbf{v}_{k}
$$

in which $\mathbf{h}\left(\mathbf{Z}_{k}\right)=-\mathbf{M}^{-1} \mathbf{F}\left[\dot{\mathbf{x}}_{k}, \mathbf{x}_{k}, \boldsymbol{\theta}\right] ; \mathbf{D}=-\mathbf{M}^{-1} \boldsymbol{\varphi} ; \mathbf{v} k$ is the measurement noise vector with zero mean and a covariance matrix $\mathbf{R}_{k}$. With proper transformation, the following revised observation equation can be derived.

$$
\boldsymbol{\Phi} \mathbf{y}_{k}=\boldsymbol{\Phi} \mathbf{h}\left(\mathbf{Z}_{k}\right)+\boldsymbol{\Phi} \mathbf{v}_{k}
$$

where $\boldsymbol{\Phi}=\mathbf{I}-\mathbf{D}\left(\mathbf{D}^{\mathrm{T}} \mathbf{D}\right)^{-1} \mathbf{D}^{\mathrm{T}}$

Let $\hat{\mathbf{Z}}_{k+1 \mid k}$ be the priori estimate of state $\mathbf{Z}_{k+1}$, and linearize $\mathbf{h}\left(\mathbf{Z}_{k+1}\right)$ with respect to $\hat{\mathbf{Z}}_{k+1 \mid k}$,

$$
\mathbf{h}\left(\mathbf{Z}_{k+1}\right)=\mathbf{h}\left(\hat{\mathbf{Z}}_{k+1 \mid k}\right)+\mathbf{H}_{k+1 \mid k}\left(\mathbf{Z}_{k+1}-\hat{\mathbf{Z}}_{k+1 \mid k}\right)
$$

where $\mathbf{H}_{k+1 \mid k}=\left.\frac{\partial \mathbf{h}\left(\mathbf{Z}_{k+1}\right)}{\partial \mathbf{Z}_{k+1}}\right|_{\mathbf{Z}_{k+1}=\hat{\mathbf{z}}_{k+1 \mid k}}$.

The priori estimated state $\hat{\mathbf{Z}}_{k+1 \mid k}$ can be determined as,

$$
\hat{\mathbf{Z}}_{k+1 \mid k}=\hat{\mathbf{Z}}_{k \mid k}+\int_{k \Delta t}^{(k+1) \Delta t} \mathbf{g}\left(\hat{\mathbf{Z}}_{k \mid k}, \hat{\mathbf{f}}_{k}^{u}, k \Delta t\right) \mathrm{d} t
$$

The priori estimation error covariance matrix is calculated as follows,

$$
\mathbf{P}_{k+1 \mid k}=\mathbf{A}_{1} \mathbf{P}_{k \mid k} \mathbf{A}_{1}^{\mathrm{T}}+\mathbf{A}_{2} \mathbf{R}_{k} \mathbf{A}_{2}^{\mathrm{T}}+\Delta t \mathbf{w}_{k}^{2}
$$


where $\mathbf{A}_{1}=\left(\mathbf{I}+\Delta t \mathbf{U}_{k \mid k}-\Delta t\left[\begin{array}{c}\mathbf{0} \\ (\mathbf{I}-\boldsymbol{\Phi}) \mathbf{H}_{k \mid k} \\ \mathbf{0}\end{array}\right]\right) ; \mathbf{A}_{2}=\left[\begin{array}{c}\mathbf{0} \\ -\Delta t(\mathbf{I}-\boldsymbol{\Phi}) \\ \mathbf{0}\end{array}\right] ; \mathbf{U}_{k \mid k}=\left.\frac{\partial \mathbf{u}\left(\mathbf{Z}_{k}, \mathbf{f}_{u}^{k}, t_{k}\right)}{\partial \mathbf{Z}_{k}}\right|_{\substack{\mathbf{Z}_{k}=\hat{\boldsymbol{k}}_{k \mid k} \\ \mathbf{f}_{k}^{k}=\hat{\mathbf{f}}_{k}^{k}}}$

The posteriori estimated state $\hat{\mathbf{Z}}_{k+1 \mid k+1}$ can be given as,

$$
\hat{\mathbf{Z}}_{k+1 \mid k+1}=\hat{\mathbf{Z}}_{k+1 \mid k}+\mathbf{G}_{k+1}\left[\boldsymbol{\Phi} \mathbf{y}_{k+1}-\boldsymbol{\Phi} \mathbf{h}\left(\hat{\mathbf{Z}}_{k+1 \mid k}\right)\right]
$$

in which $\mathbf{G}_{k+1}$ denotes the EKF gain matrix at the $(k+1)$-th time step.

$$
\mathbf{G}_{k+1}=\mathbf{P}_{k+1 \mid k} \mathbf{H}_{k+1 \mid k}^{\mathrm{T}} \boldsymbol{\Phi}^{\mathrm{T}}\left[\boldsymbol{\Phi}\left(\mathbf{H}_{k+1 \mid k} \mathbf{P}_{k+1 \mid k} \mathbf{H}_{k+1 \mid k}^{\mathrm{T}}+\mathbf{R}_{k+1}\right) \boldsymbol{\Phi}^{\mathrm{T}}\right]^{-1}
$$

Then, the posteriori estimation error covariance matrix can be obtained as

$$
\mathbf{P}_{k+1 \mid k+1}=\left(\mathbf{I}-\mathbf{G}_{k+1} \boldsymbol{\Phi} \mathbf{H}_{k+1 \mid k}\right) \mathbf{P}_{k+1 \mid k}\left(\mathbf{I}-\mathbf{G}_{k+1} \boldsymbol{\Phi} \mathbf{H}_{k+1 \mid k}\right)^{\mathrm{T}}+\mathbf{G}_{k+1} \boldsymbol{\Phi} \mathbf{R}_{k+1} \boldsymbol{\Phi}^{\mathrm{T}} \mathbf{G}_{k+1}^{\mathrm{T}}
$$

Finally, the unknown excitation is obtained by LSE as follows,

$$
\hat{\mathbf{f}}_{k+1}^{u}=\left(\mathbf{D}^{\mathrm{T}} \mathbf{D}\right)^{-1} \mathbf{D}^{\mathrm{T}}\left[\mathbf{h}\left(\hat{\mathbf{Z}}_{k+1 \mid k+1}\right)-\mathbf{y}_{k+1}\right]
$$

\subsection{The proposed OEKF-UI approach}

As shown in section 2.1, the parameters to be identified are time-invariant leading to $\dot{\boldsymbol{\theta}}(t)=0$. Thus, the aforementioned procedure cannot effectively capture the time-varying properties of structural parameters. In order to track the variation of structural parameters, an online tracking matrix (OTM) expressed as $\boldsymbol{\Lambda}_{k+1}$ is introduced in this study. $\boldsymbol{\Lambda}_{k+1}$ is a diagonal matrix with the dimension of $(2 n+m) \times(2 n+m)$, where $n$ and $m$ are the number of DOFs and unknown parameters, respectively. The first $2 n$ diagonal elements of $\boldsymbol{\Lambda}_{k+1}$ associated with displacement and velocity responses are set to be 1.0, whereas the remaining $m$ diagonal elements associated with the unknown parameters are denoted by $\lambda_{k+1}^{1}, \lambda_{k+1}^{2}, \ldots, \lambda_{k+1}^{m}$. If the values of structural parameters vary at the time instant $t_{k+1}$, the values of $\lambda_{k+1}^{i}(i=1,2, \ldots, m)$ will be changed accordingly. Based on this defined OTM, the prior estimation error $\boldsymbol{\varepsilon}_{k+1 \mid k}$ at $t_{k+1}$ can be expresses as $\boldsymbol{\varepsilon}_{k+1 \mid k}=\boldsymbol{\Lambda}_{k+1}\left(\mathbf{Z}_{k+1}-\hat{\mathbf{Z}}_{k+1 \mid k}\right)$. Then, the priori estimation error covariance matrix shown in Eq. (8) can be rewritten as

$$
\mathbf{P}_{k+1 \mid k}=\boldsymbol{\Lambda}_{k+1}\left[\mathbf{A}_{1} \mathbf{P}_{k \mid k} \mathbf{A}_{1}^{\mathrm{T}}\right] \boldsymbol{\Lambda}_{k+1}^{\mathrm{T}}+\mathbf{A}_{2} \mathbf{R}_{k} \mathbf{A}_{2}^{\mathrm{T}}+\Delta t \mathbf{Q}_{k}^{2}
$$


In general, the posteriori estimation error $\boldsymbol{\varepsilon}_{k+1 \mid k+1}$ at $t_{k+1}$ is defined as $\boldsymbol{\varepsilon}_{k+1 \mid k+1}=\mathbf{Z}_{k+1}-\hat{\mathbf{Z}}_{k+1 \mid k+1}$. Based on Eq. (5), Eq. (6) and Eq. (9), such posteriori estimation error can be calculated as

$$
\begin{aligned}
& \boldsymbol{\varepsilon}_{k+1 \mid k+1}=\mathbf{Z}_{k+1}-\hat{\mathbf{Z}}_{k+1 \mid k+1} \\
& =\mathbf{Z}_{k+1}-\hat{\mathbf{Z}}_{k+1 \mid k}-\mathbf{G}_{k+1}\left[\boldsymbol{\Phi}\left(\mathbf{Z}_{k+1}\right)+\boldsymbol{\Phi} \mathbf{v}_{k+1}-\boldsymbol{\Phi} \mathbf{h}\left(\hat{\mathbf{Z}}_{k+1 \mid k}\right)\right] \\
& =\mathbf{Z}_{k+1}-\hat{\mathbf{Z}}_{k+1 \mid k}-\mathbf{G}_{k+1}\left[\boldsymbol{\Phi} \mathbf{H}_{k+1 \mid k}\left(\mathbf{Z}_{k+1}-\hat{\mathbf{Z}}_{k+1 \mid k}\right)+\Phi \mathbf{V}_{k+1}\right] \\
& =\left(\mathbf{I}-\mathbf{G}_{k+1} \boldsymbol{\Phi} \mathbf{H}_{k+1 \mid k}\right) \Lambda_{k+1} \boldsymbol{\varepsilon}_{k+1 \mid k}-\mathbf{G}_{k+1} \boldsymbol{\Phi} \mathbf{v}_{k+1}
\end{aligned}
$$

Then, the error covariance matrix shown in Eq. (11) can be rearranged as,

$$
\mathbf{P}_{k+1 \mid k+1}=\left(\mathbf{I}-\mathbf{G}_{k+1} \boldsymbol{\Phi} \mathbf{H}_{k+1 \mid k}\right) \Lambda_{k+1} \mathbf{P}_{k+1 \mid k} \Lambda_{k+1}^{\mathrm{T}}\left(\mathbf{I}-\mathbf{G}_{k+1} \boldsymbol{\Phi} \mathbf{H}_{k+1 \mid k}\right)^{\mathrm{T}}+\mathbf{G}_{k+1} \boldsymbol{\Phi} \mathbf{R}_{k+1} \boldsymbol{\Phi}^{\mathrm{T}} \mathbf{G}_{k+1}^{\mathrm{T}}
$$

Accordingly, the gain matrix shown in Eq. (10) can be changed to

$$
\mathbf{G}_{k+1}=\boldsymbol{\Lambda}_{k+1} \mathbf{P}_{k+1 \mid k} \boldsymbol{\Lambda}_{k+1}^{\mathrm{T}} \mathbf{H}_{k+1 \mid k}^{\mathrm{T}} \boldsymbol{\Phi}^{\mathrm{T}}\left[\boldsymbol{\Phi}\left(\mathbf{H}_{k+1 \mid k} \boldsymbol{\Lambda}_{k+1} \mathbf{P}_{k+1 \mid k} \boldsymbol{\Lambda}_{k+1}^{\mathrm{T}} \mathbf{H}_{k+1 \mid k}^{\mathrm{T}}+\mathbf{R}_{k+1}\right) \boldsymbol{\Phi}^{\mathrm{T}}\right]^{-1}
$$

Thus, the proposed OEKF-UI approach is composed of three parts, saying time update by Eq (7) and Eq. (13), measurement update by Eq. (9), Eq. (15) and Eq. (16), as well as force identification by Eq. (12). It can be found that the key point of the implementation of the proposed approach is the determination of $\boldsymbol{\Lambda}_{k+1}$. In order to calculate OTM, the following derivation is conducted and an objective function is defined.

Based on Eq. (5), the predicted error vector $\mathbf{r}_{k+1}$ and residual error vector $\overline{\mathbf{r}}_{k+1}$ can be calculated as

$$
\begin{gathered}
\mathbf{r}_{k+1}=\boldsymbol{\Phi} \mathbf{y}_{k+1}-\boldsymbol{\Phi} \mathbf{h}\left(\hat{\mathbf{Z}}_{k+1 \mid k}, t_{k+1}\right) \\
\overline{\mathbf{r}}_{k+1}=\boldsymbol{\Phi} \mathbf{y}_{k+1}-\boldsymbol{\Phi} \mathbf{h}\left(\hat{\mathbf{Z}}_{k+1 \mid k+1}, t_{k+1}\right)
\end{gathered}
$$

Using Eq. (6) and Eq. (9), the residual error vector can be rearranged,

$$
\begin{aligned}
& \overline{\mathbf{r}}_{k+1}=\boldsymbol{\Phi}\left(\mathbf{y}_{k+1}-\mathbf{h}\left(\hat{\mathbf{Z}}_{k+1 \mid k+1}, t_{k+1}\right)\right) \\
& =\boldsymbol{\Phi}\left(\mathbf{y}_{k+1}-\mathbf{h}\left(\hat{\mathbf{Z}}_{k+1 \mid k}, t_{k+1}\right)-\mathbf{H}_{k+1 \mid k}\left(\hat{\mathbf{Z}}_{k+1 \mid k+1}-\hat{\mathbf{Z}}_{k+1 \mid k}\right)\right) \\
& =\boldsymbol{\Phi}\left(\mathbf{y}_{k+1}-\mathbf{h}\left(\hat{\mathbf{Z}}_{k+1 \mid k}, t_{k+1}\right)-\mathbf{H}_{k+1 \mid k} \mathbf{G}_{k+1} \boldsymbol{\Phi}\left[\mathbf{y}_{k+1}-\mathbf{h}\left(\hat{\mathbf{Z}}_{k+1 \mid k}, t_{k+1}\right)\right]\right) \\
& =\left[\mathbf{I}-\boldsymbol{\Phi} \mathbf{H}_{k+1 \mid k} \mathbf{G}_{k+1}\right] \mathbf{r}_{k+1}
\end{aligned}
$$

Taking expectation values of the residual error vector, one can obtain 


$$
E\left(\overline{\mathbf{r}}_{k+1} \overline{\mathbf{r}}_{k+1}^{\mathrm{T}}\right)=\left[\mathbf{I}-\boldsymbol{\Phi} \mathbf{H}_{k+1 \mid k} \mathbf{G}_{k+1}\right] E\left(\mathbf{r}_{k+1} \mathbf{r}_{k+1}^{\mathrm{T}}\right)\left[\mathbf{I}-\boldsymbol{\Phi} \mathbf{H}_{k+1 \mid k} \mathbf{G}_{k+1}\right]^{\mathrm{T}}
$$

Based on Eq. (5) and the definition of the covariance matrix, it can be found that the leftside-of Eq. (20) is equal to $\boldsymbol{\Phi} \mathbf{R}_{k+1} \boldsymbol{\Phi}^{\mathrm{T}}$. Here, the covariance matrix of predicted error vector can be defined as $E\left(\mathbf{r}_{k+1} \mathbf{r}_{k+1}^{\mathrm{T}}\right)=\mathbf{V}_{k+1}$. Then, with the aid of Eq. (10), the following equation can be obtained,

$$
\mathbf{V}_{k+1}=\Upsilon_{1} \Upsilon_{2} \mathbf{\Phi} \mathbf{R}_{k+1} \boldsymbol{\Phi}^{\mathrm{T}}\left(\Upsilon_{1} \Upsilon_{2}\right)^{\mathrm{T}}
$$

where $\Upsilon_{1}=\boldsymbol{\Phi}\left(\mathbf{H}_{k+1 \mid k} \mathbf{P}_{k+1 \mid k} \mathbf{H}_{k+1 \mid k}^{\mathrm{T}}+\mathbf{R}_{k+1}\right) \boldsymbol{\Phi}^{\mathrm{T}}, \Upsilon_{2}=\left(\boldsymbol{\Phi} \mathbf{R}_{k+1} \boldsymbol{\Phi}^{\mathrm{T}}\right)^{-1}$.

It can be seen that $\mathbf{P}_{k+1 \mid k}$ in Eq. (21) contains $\boldsymbol{\Lambda}_{k+1}$. Thus, the OTM can be determined by solving Eq. (21). However, Eq. (21) is highly nonlinear and difficult to be solved directly. Instead, an optimization procedure is employed herein for the determination of $\boldsymbol{\Lambda}_{k+1}$. An objective function is defined as,

$$
\Omega_{k+1}=\sum_{i=1}^{m}\left|\frac{\theta_{k+1}^{i}-\theta_{k}^{i}}{\theta_{k}^{i}}\right|
$$

where $\hat{\theta}_{k+1}^{i}$ and $\hat{\theta}_{k}^{i}$ denote the $i$-th identified parameters at $t_{k+1}$ and $t_{k}$, respectively.

Then, the purpose of determining $\boldsymbol{\Lambda}_{k+1}$ can be transformed into an optimization issue by minimizing the objective function in Eq. (22) under the following constraint,

$$
\left\|\mathbf{V}_{k+1}-\Upsilon_{1} \Upsilon_{2} \boldsymbol{\Phi} \mathbf{R}_{k+1} \boldsymbol{\Phi}^{\mathrm{T}}\left(\Upsilon_{1} \Upsilon_{2}\right)^{\mathrm{T}}\right\| \leq \delta
$$

where $\|\cdot\|$ denotes Frobenius norm; $\delta$ is a small positive constant number. In this study, $\delta=$ 0.01 is used in the numerical examples. The function 'FMINCON' in MATLAB is a very convenient tool to obtain the optimal solution of $\boldsymbol{\Lambda}_{k+1}$. To effectively conduct this optimization procedure, a reasonable value of $\mathbf{V}_{k+1}$ matrix calculated at time step of $t_{k+1}$ is required. The followings are how to determine such matrix.

Referring to the method introduced by Yang et al. [36], a special case that the diagonal elements of $\boldsymbol{\Lambda}_{k+1}$ are identical is first considered, i.e., $\boldsymbol{\Lambda}_{k+1}=\zeta_{k+1}^{1 / 2} \mathbf{I}_{2 n+m}$ where $\zeta_{k+1}$ is the tracking coefficient to be determined. Then, Eq. (13) can be expressed as,

$$
\mathbf{P}_{k+1 \mid k}=\zeta_{k+1}\left[\mathbf{A}_{1} \mathbf{P}_{k \mid k} \mathbf{A}_{1}^{\mathrm{T}}\right]+\mathbf{A}_{2} \mathbf{R}_{k} \mathbf{A}_{2}^{\mathrm{T}}+\Delta t \mathbf{Q}_{k}^{2}
$$

Substituting Eq. (24) into Eq. (21), the following equation can be obtained

$$
\mathbf{V}_{k+1}=\left(\zeta_{k+1} \mathbf{T}_{1} \mathbf{T}_{3}^{-1}+\mathbf{T}_{2} \mathbf{T}_{3}^{-1}+\mathbf{T}_{3} \mathbf{T}_{3}^{-1}\right) \mathbf{T}_{3}\left(\zeta_{k+1} \mathbf{T}_{1} \mathbf{T}_{3}^{-1}+\mathbf{T}_{2} \mathbf{T}_{3}^{-1}+\mathbf{T}_{3} \mathbf{T}_{3}^{-1}\right)^{\mathrm{T}}
$$


where $\quad \mathbf{T}_{1}=\boldsymbol{\Phi} \mathbf{H}_{k+1 \mid k} \mathbf{A}_{1} \mathbf{P}_{k \mid k} \mathbf{A}_{1}^{\mathrm{T}} \mathbf{H}_{k+1 \mid k}^{\mathrm{T}} \boldsymbol{\Phi}^{\mathrm{T}} ; \mathbf{T}_{2}=\boldsymbol{\Phi} \mathbf{H}_{k+1 \mid k}\left(\mathbf{A}_{2} \mathbf{R}_{k} \mathbf{A}_{2}^{T}+\Delta t \mathbf{Q}_{k}^{2}\right) \mathbf{H}_{k+1 \mid k}^{\mathrm{T}} \boldsymbol{\Phi}^{\mathrm{T}} ;$ $\mathbf{T}_{3}=\boldsymbol{\Phi} \mathbf{R}_{k+1} \boldsymbol{\Phi}^{\mathrm{T}}$

Taking the trace on both sides of Eq. (25), one can obtain,

$$
\begin{aligned}
\operatorname{tr}\left\{\mathbf{V}_{k+1}\right\}= & \zeta_{k+1}^{2} \operatorname{tr}\left\{\mathbf{T}_{1} \mathbf{T}_{3}^{-1} \mathbf{T}_{3}\left(\mathbf{T}_{1} \mathbf{T}_{3}^{-1}\right)^{\mathrm{T}}\right\}+\zeta_{k+1} \operatorname{tr}\left\{\left(\mathbf{T}_{2} \mathbf{T}_{3}^{-1}+\mathbf{T}_{3} \mathbf{T}_{3}^{-1}\right) \mathbf{T}_{3}\left(\mathbf{T}_{1} \mathbf{T}_{3}^{-1}\right)^{\mathrm{T}}\right. \\
& \left.+\mathbf{T}_{1} \mathbf{T}_{3}^{-1} \mathbf{T}_{3}\left(\mathbf{T}_{2} \mathbf{T}_{3}^{-1}+\mathbf{T}_{3} \mathbf{T}_{3}^{-1}\right)^{\mathrm{T}}\right\}+\operatorname{tr}\left\{\left(\mathbf{T}_{2} \mathbf{T}_{3}^{-1}+\mathbf{T}_{3} \mathbf{T}_{3}^{-1}\right) \mathbf{T}_{3}\left(\mathbf{T}_{2} \mathbf{T}_{3}^{-1}+\mathbf{T}_{3} \mathbf{T}_{3}^{-1}\right)^{\mathrm{T}}\right\}
\end{aligned}
$$

By solving Eq. (26), $\zeta_{k+1}$ can be calculated as,

$$
\zeta_{k+1}=\left(-\mathrm{T}_{b}+\sqrt{\mathrm{T}_{b}^{2}-4 \mathrm{~T}_{a} \mathrm{~T}_{c}}\right) / 2 \mathrm{~T}_{a} \quad\left(\bar{\lambda}_{k+1} \geq 1\right)
$$

where

$$
\begin{aligned}
& \mathrm{T}_{a}=\operatorname{tr}\left\{\mathbf{T}_{1} \mathbf{T}_{3}^{-1} \mathbf{T}_{3}\left(\mathbf{T}_{1} \mathbf{T}_{3}^{-1}\right)^{\mathrm{T}}\right\} ; \mathrm{T}_{b}=\operatorname{tr}\left\{\left(\mathbf{T}_{2} \mathbf{T}_{3}^{-1}+\mathbf{T}_{3} \mathbf{T}_{3}^{-1}\right) \mathbf{T}_{3}\left(\mathbf{T}_{1} \mathbf{T}_{3}^{-1}\right)^{\mathrm{T}}+\left(\mathbf{T}_{1} \mathbf{T}_{3}\right) \mathbf{T}_{3}\left(\mathbf{T}_{2} \mathbf{T}_{3}^{-1}+\mathbf{T}_{3} \mathbf{T}_{3}^{-1}\right)^{\mathrm{T}}\right\} ; \\
& \mathrm{T}_{c}=\operatorname{tr}\left\{\left(\mathbf{T}_{2} \mathbf{T}_{3}^{-1}+\mathbf{T}_{3} \mathbf{T}_{3}^{-1}\right) \mathbf{T}_{3}\left(\mathbf{T}_{2} \mathbf{T}_{3}^{-1}+\mathbf{T}_{3} \mathbf{T}_{3}^{-1}\right)^{\mathrm{T}}\right\}-\operatorname{tr}\left\{\mathbf{V}_{k+1}\right\} .
\end{aligned}
$$

To determine the value of $\boldsymbol{\Lambda}_{k+1}$ at the time instant $t_{k+1}$, the covariance matrix $\mathbf{V}_{k+1}$ should also be evaluated for the implementation of the aforementioned optimization procedure. After calculating the value of $\zeta_{k+1}$ by Eqs. (24-27), the following recursive estimation for $\mathrm{V}_{k+1}$ can be conducted [36],

$$
\mathbf{V}_{k+1}=\mathbf{K}_{1, k+1} / \mathbf{K}_{2, k+1}
$$

where $\mathbf{K}_{1, k+1}=\mathbf{r}_{k+1} \mathbf{r}_{k+1}^{\mathrm{T}}+v \mathbf{K}_{1, k} / \zeta_{k} ; \mathbf{K}_{2, k+1}=1+v \mathbf{K}_{2, k} / \zeta_{k} ; v$ is a coefficient set to be 0.95 in this study.

In summary, similar to the framework of EKF technique, the proposed OEKF-UI approach in this paper contains time update procedure by Eq (7) and Eq. (13), measurement update procedure by Eq. (9), Eq. (15) and Eq. (16). Moreover, Eq. (12) is derived to identify the unknown inputs. To track the time-varying parameters, the optimization problem shown in Eqs. (22-23) is solved for the determination of OTM. The flowchart of the proposed approach is shown in Fig. 1 for ease of understanding. The effectiveness of the proposed approach is demonstrated via several linear and nonlinear examples in the following section.

\section{Numerical investigation}

In this section, a 4-story shear-type linear structure with and without nonlinear components are considered. The mass and stiffness of each floor are set to be $300 \mathrm{~kg}$ and 180 
$\mathrm{kN} / \mathrm{m}$, respectively. To mimic structural damage, the structural stiffness is assumed to be changed abruptly at some time. The acceleration responses with the sampling frequency of $1000 \mathrm{~Hz}$ are used for the identification of such time-variant parameters and unknown inputs. From a practical viewpoint, the measured acceleration responses are simulated by the theoretically computed quantities superimposed with the corresponding noise process with $5 \%$ noise to signal ratio.

\subsection{Four-story linear building with multiple abrupt damages}

In this example, a random excitation is applied on the top floor of the structure. After the calculation of structural responses by state space method, this excitation is assumed to be unknown. The Rayleigh damping model is adopted, i.e., $\mathbf{C}=\alpha \mathbf{M}+\beta \mathbf{K}$. The damping coefficients $\alpha$ and $\beta$ are set to be 0.3788 and 0.0018 , respectively. To mimic time-varying multiple damages, the abrupt changes of structural stiffness of the $2^{\text {nd }}$ and $4^{\text {th }}$ floor are considered as follow,

$$
k_{2}=\left\{\begin{array}{ll}
180(\mathrm{kN} / \mathrm{m}) & 0 \mathrm{~s} \leq t<5 \mathrm{~s} \\
144(\mathrm{kN} / \mathrm{m}) & 5 \mathrm{~s} \leq t<7 \mathrm{~s} ; \\
108(\mathrm{kN} / \mathrm{m}) & 7 \mathrm{~s} \leq t<10 \mathrm{~s}
\end{array} \quad k_{4}= \begin{cases}180(\mathrm{kN} / \mathrm{m}) & 0 \mathrm{~s} \leq t<7 \mathrm{~s} \\
162(\mathrm{kN} / \mathrm{m}) & 7 \mathrm{~s} \leq t<10 \mathrm{~s}\end{cases}\right.
$$

The quantities to be identified include the damping coefficients, stiffness parameters, and unknown excitation. The initial values of the structural parameters are assumed to be $50 \%$ of the actual ones.

By using the proposed approach, the structural parameters can be identified as shown in Fig. 2. Only the stiffness parameters are plotted in Fig. 2 as examples. The identification results without using OTM are given in Fig. 2 as well. It can be seen that, although oscillation exists at the beginning, the identified results can still converge to those real ones after few seconds. As compared with the results without OTM, it is obvious that the abrupt changes of structural stiffness can be accurately captured by the proposed approach.

Besides the identification of structural parameters, the unknown force applied to the top floor can also be identified. Fig. 3 gives the time series of the identified excitation. Only the time segment from $7.0 \mathrm{~s}$ to $7.1 \mathrm{~s}$ is plotted in Fig. 3 for clarity of comparison. It is clear that the identified inputs are close to the actual ones.

\subsection{Building structure equipped with Duffing model}

Nonlinearity exists widely in the engineering structures. To investigate the proposed approach for the identification of time-varying parameters of nonlinear system, a building 
structure with slight nonlinear phenomenon is first considered in this example. The 4-story building model introduced before is employed in this section. The nonlinear Duffing model expressed by Eq. (30) is added on each floor and used to generate nonlinear restoring force $(\mathrm{NRF})$.

$$
\mathbf{N R F}=\mathbf{K}_{d} \Delta \mathbf{x}^{3}(t)
$$

where $\mathbf{K}_{d}$ is a diagonal coefficient matrix; $\Delta \mathbf{x}$ is inter-story drift. Here, the diagonal elements of $\mathbf{K}_{d}$ are set to be $k_{d i}=1 \times 10^{7} \mathrm{~N} / \mathrm{m}^{3}(i=1,2,3)$ and $k_{d 4}=0.5 \times 10^{7} \mathrm{~N} / \mathrm{m}^{3}$.

The El-Centro earthquake with a peak ground acceleration (PGA) of $3.4 \mathrm{~m} / \mathrm{s}^{2}$ is employed as external excitation. The corresponding nonlinear responses are computed by Runge-Kunta method with the time interval of $0.001 \mathrm{~s}$. A dual abrupt damage is considered in this example, saying the stiffness of the $1^{\text {st }}$ and $3^{\text {rd }}$ story are reduced sharply by $30 \%$ and $20 \%$ at $t_{k}=5 \mathrm{~s}$, respectively. The quantities to be identified include the coefficients of structural damping, stiffness and Duffing model, as well as unknown ground motion. Similarly, the initial values of these coefficients are all assumed to be $50 \%$ of the actual ones.

Based on the measured acceleration responses, the coefficients can be identified by the proposed approach as depicted in Fig. 4. The results without OTM are also given in Fig. 4 for comparison. Obviously, the abrupt changes can be effectively tracked with the usage of OTM, whereas unsatisfactory results will be obtained without OTM. Figure 5 gives the results of the identified unknown seismic input. It can be seen that the identified inputs have a good agreement with the actual ones.

\subsection{Building structure equipped with Bouc-Wen model}

To further verify the feasibility of the proposed approach for identifying the parameters of structures with highly nonlinear properties, the aforementioned building structure equipped with Bouc-Wen model on each floor is considered herein. Then, the equation of motion shown in Eq. (1) can be re-written as,

$$
\mathbf{M} \ddot{\mathbf{x}}(t)+\mathbf{C} \dot{\mathbf{x}}(t)+\mathbf{K z}(t)=\boldsymbol{\varphi}^{u} \mathbf{f}^{u}(t)
$$

where $\mathbf{M}, \mathbf{K}$, and $\mathbf{C}$ and structural parameters as introduced before. In this example, viscous damping rather than Rayleigh damping is used for the construction of damping matrix. The damping coefficients are set to be $800 \mathrm{~N} \cdot \mathrm{s} / \mathrm{m}$ for each story. $\mathbf{z}(t)$ is hysteretic component given as,

$$
\dot{z}_{i}=\dot{x}_{i}-\dot{x}_{i-1}-\beta\left|\dot{x}_{i}-\dot{x}_{i-1}\right|\left|z_{i}\right|^{\mu_{i}-1}-\gamma_{i}\left(\dot{x}_{i}-\dot{x}_{i-1}\right)\left|z_{i}\right|^{\mu_{i}}
$$


where $\beta_{i}, \gamma_{i}$ and $\mu_{i}$ are the coefficients of Bouc-Wen model on the $i$-th floor. Here, the values of $\beta, \gamma$ and $\mu$ for each floor are set to be 3.5, 2.5 and 2, respectively.

A random excitation is applied on the top floor of the building. The hysteretic responses are then computed by means of Runge-Kutta method with a time interval of $0.001 \mathrm{~s}$. A severe damage is assumed to occur at $t=5 \mathrm{~s}$ on the first floor. The stiffness of this floor $k_{1}$ suddenly reduces from $180 \mathrm{kN} / \mathrm{m}$ to $90 \mathrm{kN} / \mathrm{m}$ resulting in $50 \%$ stiffness degradation, and viscous damping $c_{1}$ reduces from $800 \mathrm{~N} \mathrm{~s} / \mathrm{m}$ to $400 \mathrm{~N} \mathrm{~s} / \mathrm{m}$ at the same time. Besides, the Bouc-Wen model coefficient $\gamma_{1}$ is also decreased $50 \%$. The quantities to be identified include the coefficients of structural damping, stiffness and Bouc-Wen model, as well as unknown external force. Similarly, the initial values of these coefficients are $50 \%$ of the actual ones. The time series of the identified parameters of the $1^{\text {st }}$ and $2^{\text {nd }}$ floor are plotted in Fig. 6 as examples. Moreover, the time-varying parameters identified by other methods [24] are shown in Fig. 6 for comparison. It is obvious that in the case of severe damage occurred, the identified parameters without using OTM are not correct at all. The performance of the proposed OEKF-UI is comparable with Yang's method. Both of them are capable of identifying the suddenly-changed parameters with acceptable accuracy.

The unknown external excitation is identified and shown in Fig. 7. The time segment from $7 \mathrm{~s}$ to $7.1 \mathrm{~s}$ is plotted for clarity of comparison. It can be seen that the identified inputs match with the actual ones well.

\section{Conclusions}

In this paper, in order to effectively identify time-varying structural parameters without prior knowledge of external excitation, an online EKF-UI (OEKF-UI) approach is proposed. With the aid of projection matrix, a revised observation equation is derived. An online tracking matrix (OTM) is defined and used for tracking the time-variant properties of parameters. An optimization procedure is performed at each time step for the determination of OTM. Then, based on the principle of EKF, the recursive solution of structural states including the time-varying parameters can be analytically derived. Finally, using the estimated structural states, the unknown inputs can be identified by means of least-squares estimation (LSE) at the same time-step. Several linear and nonlinear numerical examples are used to validate the effectiveness of the proposed approach. Results show that the proposed OEKF-UI approach is capable of satisfactorily identifying structural parameters and unknown inputs even in the case of sudden severe damage. 


\section{Acknowledgement}

The authors gratefully acknowledge the financial support from the National Key Research and Development Program of China through grant No. 2019YFC1511101. The support from Natural Science Foundation of Hunan Province (No. 2021JJ30110) is also greatly appreciated.

\section{Conflict of Interest}

The authors declare that they have no conflict of interest.

\section{Data Availability Statements}

The datasets generated during and/or analyzed during the current study are available from the corresponding author on reasonable request.

\section{References}

1. Xu, Y.L., He, J.: Smart Civil Structures, CRC Press, Boca Raton (2017)

2. Das, S., Saha, P.: Structural health monitoring techniques implemented on IASC-ASCE benchmark problem: a review. J. Civ. Struct. Health Monit. 8, 689-718 (2018)

3. Quaranta, G., Lacarbonara, W., Masri, S.F.: A review on computational intelligence for identification of nonlinear dynamical systems. Nonlinear Dyn. 99(2), 1709-1761 (2020)

4. Yun, C.B., Shinozuka, M.: Identification of nonlinear structural dynamic systems. J. Struct. Mech. 8(2) 187203 (1980)

5. Hoshiya, M., Saito, E.: Structural identification by extended Kalman filter. J. Struct. Mech. 110(12), 17571770 (1984)

6. Lin, J.S., Zhang, Y.: Nonlinear structural identification using extended Kalman filter. Comput. Struct. 52(4), 757-764 (1994)

7. Khalil,M., Sarkar, A., Adhikari, S.: Nonlinear filters for chaotic oscillatory systems. Nonlinear Dyn. 55(12), 113-137(2009)

8. Xu, B., Li, J., Dyke, S.J., Deng, B.C., He, J.: Nonparametric identification for hysteretic behavior modeled with a power series polynomial using EKF-WGI approach under limited acceleration and unknown mass. Int. J. Non-Linear Mech. 119, 103324 (2020)

9. Lei, Y., Jiang, Y., Xu, Z.: Structural damage detection with limited input and output measurement signals. Mech. Syst. Signal Proc. 28(5), 229-243 (2012)

10. Xu, B., He, J.: Substructural parameters and dynamic loading identification with limited observations. Smart Struct. Syst. 15(1), 169-189 (2015)

11. Pan, S., Xiao, D., Xing, S., Law, S.S., Du, P., Li, Y.: A general extended Kalman filter for simultaneous estimation of system and unknown inputs. Eng. Struct. 109, 85-98 (2016) 
12. Liu, L.J., Hua, W., Lei, Y.: Real-time simultaneous identification of structural systems and unknown inputs without collocated acceleration measurements based on MEKF-UI. Measurement. 122, 545-553 (2018)

13. Lei, Y., Yang, X., Huang, J.: Identification of model-free hysteretic forces of magnetorheological dampers embedded in buildings under unknown excitations using incomplete structural responses. Struct. Control Health Monit. 28(5), e2715 (2021)

14. Impraimakis, M., Smyth, A.W.: An unscented Kalman filter method for real time-state estimation. Mech. Syst. Signal Proc. 162, 108026 (2022)

15. Cooper, J.E., Worden, K.: On-line physical parameter estimation with adaptive forgetting factors. Mech. Syst. Signal Proc. 14(5), 705-730 (2000)

16. Yang, J.N., Lin, S.: Identification of parametric variations of structures based on least squares estimation and adaptive tracking technique. J. Eng. Mech. 131(3), 290-298 (2005)

17. Huang, Q., Xu, Y.L., Liu, H.J.: An efficient algorithm for simultaneous identification of time-varying structural parameters and unknown excitations of a building structure. Eng. Struct. 98, 29-37 (2015)

18. Su, W.C., Liu, C.Y., Huang, C.S.: Identification of instantaneous modal parameter of time-varying systems via a wavelet-based approach and its application. Comput-Aided Civ Inf. 29 (4), 279-298 (2014)

19. Wang, C., Ren, W.X., Wang, Z.C.: Time-varying physical parameter identification of shear type structure based on wavelet transform. Smart. Struct. Syst. 14(15), 831-845 (2014)

20. Wang, Z.C., Ren, W.X., Chen. G.D.: A Hilbert transform method for parameter identification of timevarying structures with observer techniques. Smart Mater. Struct. 21(10), 105007 (2012)

21. Ahmed-Ali, T., Kenne, G., Lamnabhi-Lagarrigue, F.: Identification of nonlinear systems with time-varying parameters using a sliding-neural network observer. Neurocomputing. 72(7-9), 1611-1620 (2009)

22. Loh, C.H., Lin, C.Y., Huang, C.C.: Time domain identification of frames under earthquake loadings. J. Eng. Mech. 126(7), 693-703 (2000)

23. Yang, J.N., Lin, S., Huang, H.W., Zhou, L.: An adaptive extended Kalman filter for structural damage identification. Struct. Control. Health Monit. 13(4), 849-867 (2006)

24. Yang J.N., Pan S, Huang H.: An adaptive extended Kalman filter for structural damage identifications II: unknown inputs. Struct. Control. Health Monit. 14(3), 497-521 (2007)

25. Zhou, L., Wu, S., Yang, J.N.: Experimental study of an adaptive extended Kalman filter for structural damage identification. J. Infrastruct. Syst. 14(1), 42-51 (2008)

26. Yin, Q., Zhou, L., Mu, T., Yang, J.N.: Experimental study on damage detection of base-isolated structure using adaptive extended Kalman filter. J. Theor. Appl. Mech. 51(4), 1013-1026 (2013)

27. Li, J.P., Hua, C.C., Tang, Y.G., Guan, X.P.: A time-varying forgetting factor stochastic gradient combined with Kalman filter algorithm for parameter identification of dynamic systems. Nonlinear Dyn. 78(3), 19431952 (2014)

28. Chen, S., Lu, J., Lei, Y.: Identification of time-varying systems with partial acceleration measurements by synthesis of wavelet decomposition and Kalman filter. Adv. Mech. Eng. 12(6), 1-11 (2014)

29. Lei, Y., Yang, N.: Simultaneous identification of structural time-varying physical parameters and unknown excitations using partial measurements. Eng. Struct. 214, 110672 (2020)

30. Yang, N., Li, J., Lei, Y., Hao, H.: Identification of time-varying nonlinear structural physical parameters by integrated WMA and UKF/UKF-UI. Nonlinear Dyn. 106(1), 681-706 (2021) 
31. Yang, Y.H., Nagayama, T., Xue, K.: Structure system estimation under seismic excitation with an adaptive extended Kalman filter. J. Sound Vibr. 489, 115690 (2020)

32. Huang, Y., Yu, J.Q., Beck, J.L., Zhu, H.P., Li, H.: Novel sparseness-inducing dual Kalman filter and its application to tracking time-varying spatially-sparse structural stiffness changes and inputs. Comput. Meth. Appl. Mech. Eng. 372, 113411 (2020)

33. Lei, Y., Lu, J.B., Huang, J.S.: Integration of identification and vibration control of time-varying structures subject to unknown seismic ground excitation. J. Vib. Control. 26(15-16), 1330-1344 (2020)

34. He, J., Zhang, X.X., Xu, B.: Identification of Structural Parameters and Unknown Inputs Based on Revised Observation Equation: Approach and Validation. Int. J. Struct. Stab. Dyn. 19(2), 1950156 (2019)

35. He, J., Qi, M.C., Hua, X.G., Chen, Z.Q., Yang, O., Cao, Z.: Substructural identification with weighted global iteration considering unknown interfacial forces and external excitation. Measurement. 180, 109537 (2021)

36. Yang, J.N., Lin, S, Zhou, L.: Identification of parametric changes for structural health monitoring using an adaptive filtering technique. Proceedings of SPIE. 5391, 389-399 (2004) 


\section{List of Figure Captions}

Fig. 1 Flowchart of the proposed approach

Fig. 2 The Identified structural parameters (linear model). (a) $k_{1}$; (b) $k_{2}$; (c) $k_{3}$; (d) $k_{4}$

Fig. 3 The identified unknown input (linear model)

Fig. 4 The identified coefficients (nonlinear Duffing system). (a) $k_{1}$; (b) $k_{3}$; (c) $k_{\mathrm{d} 1}$; (d) $k_{\mathrm{d} 2}$; (e) $\alpha$; (f) $\beta$

Fig. 5 The identified unknown seismic input (Duffing system). (a) The entire time history; (b) The segment from 4 to $8 \mathrm{~s}$

Fig. 6 The identified coefficients (Bouc-Wen model). (a) $k_{1}$; (b) $k_{2}$; (c) $\mathrm{c}_{1}$; (d) $\mathrm{c}_{2}$; (e) $\beta_{1}$; (f) $\beta_{2}$; (g) $\gamma_{1}$; (h) $\gamma_{2}$

Fig. 7 The identified unknown input (Bouc-Wen model). (a) The entire time history; (b) The segment from 7 to $7.1 \mathrm{~s}$ 
Figures

\section{Figure 1}

Flowchart of the proposed approach

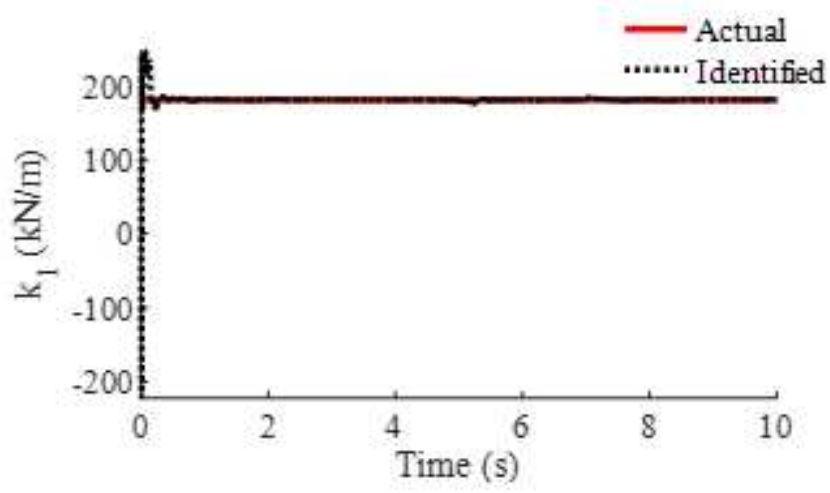

(a)

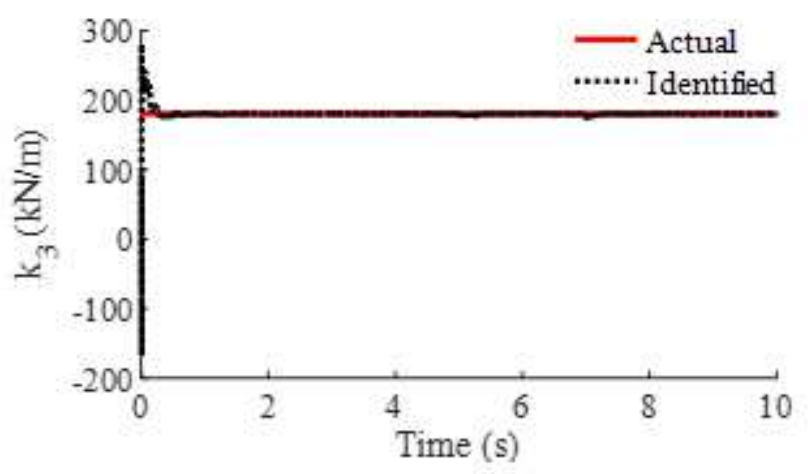

(c)

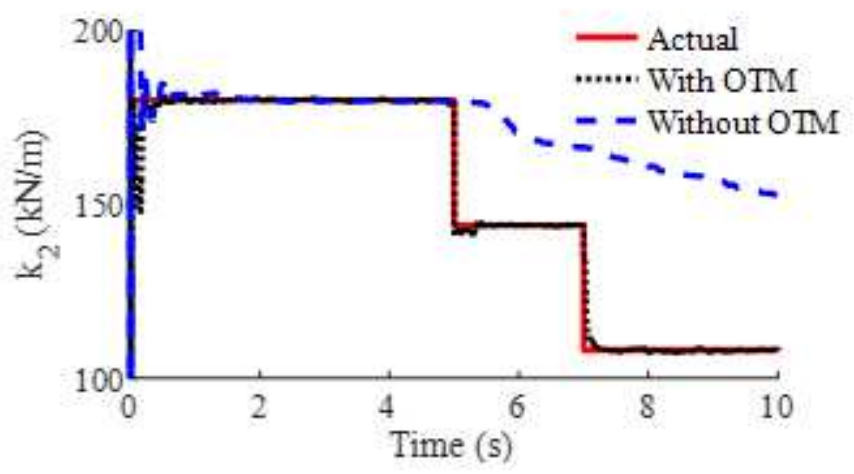

(b)

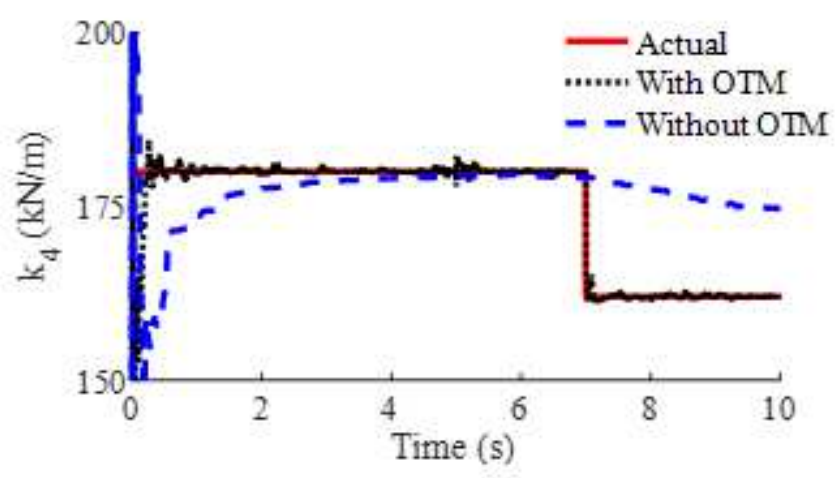

(d)

\section{Figure 2}

The Identified structural parameters (linear model). (a) k1; (b) k2; (c) k3; (d) k4

\section{Figure 3}

The identified unknown input (linear model) 


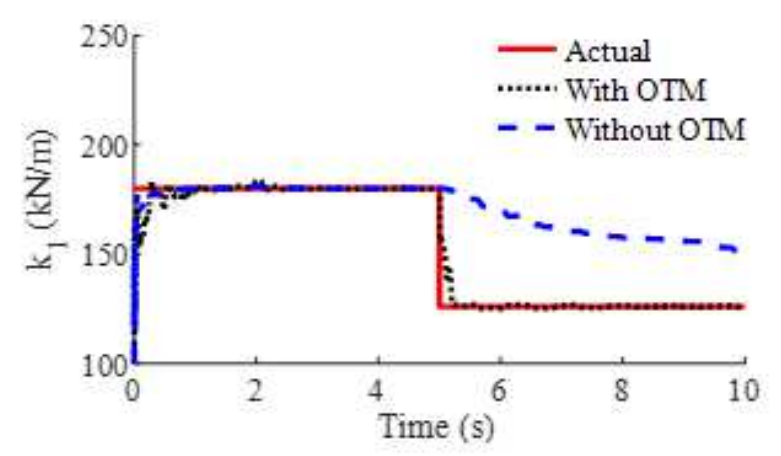

(a)

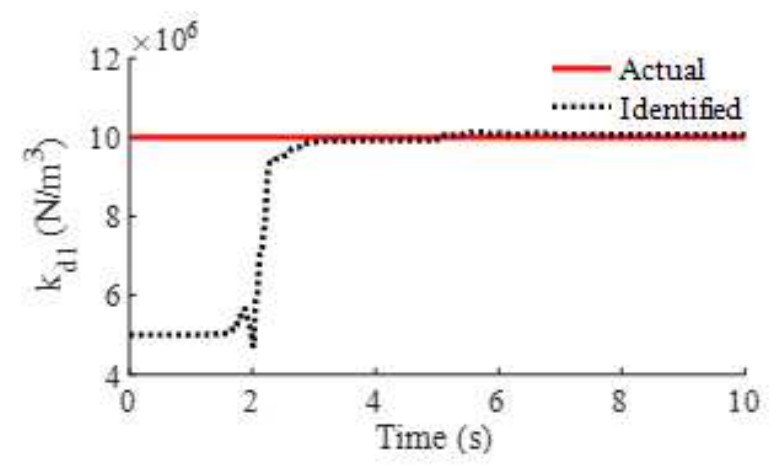

(c)

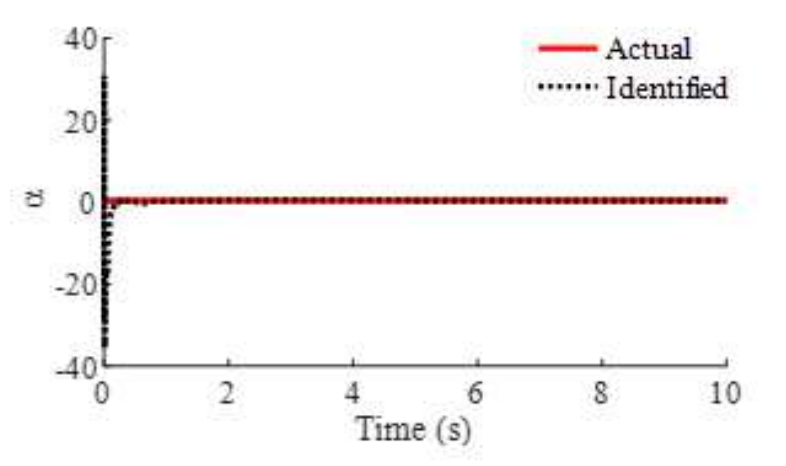

(e)

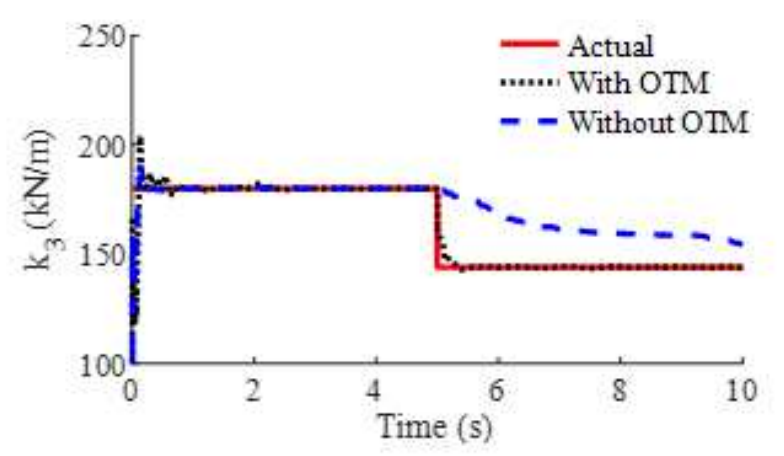

(b)

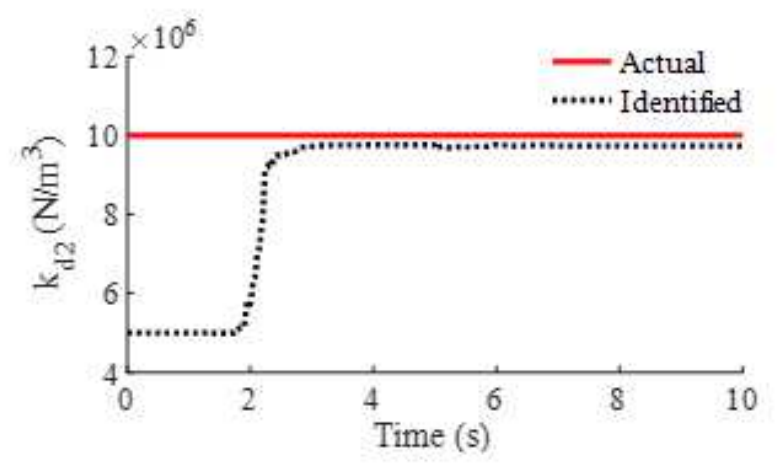

(d)

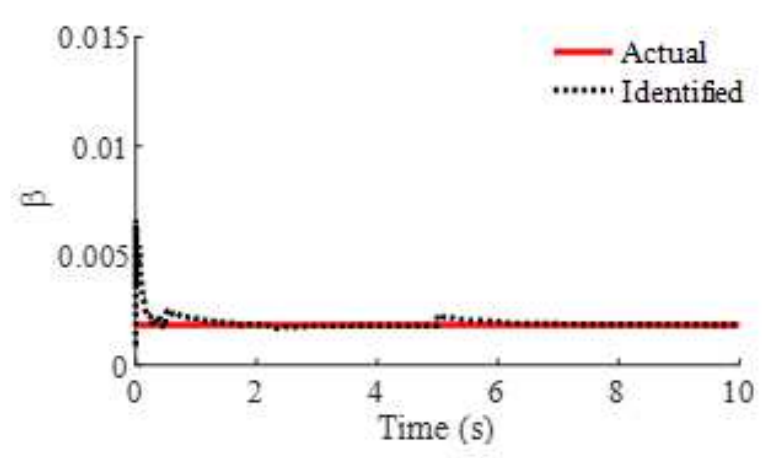

(f)

\section{Figure 4}

The identified coefficients (nonlinear Duffing system). (a) k1; (b) k3; (c) kd1; (d) kd2; (e) $a$; (f) $\beta$

\section{Figure 5}


The identified unknown seismic input (Duffing system). (a) The entire time history; (b) The segment from 4 to $8 \mathrm{~s}$

\section{Figure 6}

The identified coefficients (Bouc-Wen model). (a) k1; (b) k2; (c) c1; (d) c2 ; (e) $\beta 1$; (f) $\beta 2$; (g) $ү 1$; (h) Y2

\section{Figure 7}

The identified unknown input (Bouc-Wen model). (a) The entire time history; (b) The segment from 7 to $7.1 \mathrm{~s}$ 